\title{
Hacia una pedagogía afectiva del movimiento
}

\author{
Towards an affective pedagogy of movement
}

\author{
Silvia De Riba Mayoral \\ sderiba@gmail.com \\ Universidad de Barcelona, España \\ Beatriz Revelles Benavente \\ beatrizrevelles@ugr.es \\ Universidad de Granada, España.
}

Recibido 08/11/2018

Aceptado 08/12/2018
Investigación

\section{Resumen}

Entender la pedagogía desde el concepto del afecto permite adentrarnos en las transformaciones que los cuerpos viven dentro de los eventos de aprendizaje. En concreto, este artículo indaga sobre cómo los afectos circulan en situaciones pedagógicas de movimiento localizadas, en 3 sesiones de psicomotricidad, para ir hacia una pedagogía afectiva que explora las transiciones de los cuerpos como manera de repensar las formas de hacer en educación. La

\section{Abstract}

Moving towards an affective pedagogy means to attend the transitions between states produced in contact with the world. These transitions can transform the bodies and let them learn. An affective pedagogy of the movement explores how affect circulates in bodies in order to investigate the pedagogical sense of movement. In the same way, that vision can give new ways of understanding learning. Founded

Sugerencias para citar este artículo De Riba Mayoral, Silvia y Revelles Benavente, Beatriz (2019). Hacia una pedagogia afectiva del movimiento. Tercio Creciente, 16, págs. 7-30. https://dx.doi.org/10.17561/rtc.n16.1

DE RIBA MAYORAL, SILVIA Y REVELLES BENAVENTE, BEATRIZ. Hacia una pedagogia afectiva del movimiento. Tercio Creciente, julio 2019. $\mathrm{n}^{\circ}$ 16, pp. 7-30. https://dx.doi.org/10.17561/rtc.n16.1 
Hacia una pedagogía afectiva del movimiento / Towards an affective pedagogy of movement

https://dx.doi.org/10.17561/rtc.n16.1

metodología usada en este artículo es de una investigación basada en las artes desde una perspectiva de los nuevos materialismos. Como método, se explora el proceso de elaboración de las cartografías de investigación y cart(e) ografías que materializan el movimiento y los afectos de un momento en concreto. Este artículo entiende como resultados aquellos conocimientos surgidos en el proceso de investigación. En ellos, se puede ver como el movimiento permite circular afectos que pueden ser retenidos en la especificidad de cada cuerpo para transformar y aprender. Acercarse a una pedagogía afectiva del movimiento significa ir hacia métodos experimentales, pero localizados. De esta manera se pueden explorar estrategias pedagógicas que inviten a repensar la educación desdibujando las dualidades y las jerarquías, atender a las relaciones, a los movimientos de los cuerpos que nunca son estables y empezar a entender la necesidad de afectar y ser afectado para poder transformarse y aprender. on Arts-Based Research and a New Materialism perspective, this project explores two methods (research cartographies and cart(e)ographies) in order to investigate how movement allows the transition of affects and the new visions that this can contribute to the pedagogy field. This article interprets results as the knowledge emerged from the research process. In this sense, it can be observed that an affect produced in movement could be conserved into a body and to transform it. Furthermore, the need can be seen to attend each body specificity in the pedagogical events where the human and non-human bodies are equal participants.

To approach affective pedagogy of the movement means to use experimental but located methods in order to explore pedagogical strategies that generate specific but significant narratives to rethink pedagogy. Then, to attend affects can transform the learning as an event where bodies and its intracctions with the world are an essential part of it, allowing the becoming.

\author{
Palabras clave / Keywords \\ Afectos, cuerpo, movimiento, arte, pedagogía, educación. \\ Affect, Body, Movement, Art, Pedagogy, Education.
}

Sugerencias para citar este artículo

De Riba Mayoral, Silvia y Revelles Benavente, Beatriz (2019). Hacia una pedagogia afectiva del movimiento. Tercio Creciente, 16, págs. 7-30. https://dx.doi.org/10.17561/rtc.n16.1

DE RIBA MAYORAL, SILVIA Y REVELLES BENAVENTE, BEATRIZ. Hacia una pedagogia afectiva del movimiento. Tercio Creciente, julio 2019. ${ }^{\circ}$ 16, pp. 7-30. https://dx.doi.org/10.17561/rtc.n16.1 
Revista de Estudios en Sociedad, Artes y Gestión Cultural

www.terciocreciente.com http://revistaselectronicas.ujaen.es/index.php/RTC
Número 16

Julio 2019

Investigación
ISSN: 2340-9096

DOI: https://dx.doi.org/10.17561/rtc.

https://dx.doi.org/10.17561/rtc.n16.1

\section{Introducción.}

Este artículo parte de una conceptualización del concepto spinoziano del afecto como el tránsito entre estados que permite transformaciones que posibilitan el aprendizaje. En pedagogía, este concepto nos sirve para indagar los tránsitos que ocurren cuando los cuerpos están en movimiento. Una pedagogía afectiva del movimiento, puesto que invita a explorar estos momentos de cambio donde el cuerpo se transforma y puede ir más allá para desdibujar dicotomías y jerarquías presentes en educación, entendiendo así el aprender como un devenir. Para indagar sobre ello, es necesario atender al proceso y la transparencia (Souminen, KallioTavin y Hernández-Hernández, 2017: 105) como manera de transferir la experiencia de la investigación y abrir nuevas preguntas. Por esta razón, a continuación, detallamos los pasos seguidos en el presente estudio:

En primer lugar, exploraremos los referentes teóricos que nos abren a los afectos desde una mirada de los nuevos materialismos (van der Tuin, 2015; Rogowska-Stangret, 2017; Colman, 2014), para generar un marco teórico que nos acerca a situaciones pedagógicas de movimiento concretas. En la conformación del marco teórico, nos detendremos en la explicación de conceptos claves para entender el análisis que realizamos de la Escuela Drassanes y el centro Tariqa como ilustradores de la pedagogía afectiva del movimiento. Estos conceptos claves son la definición de afecto, cuerpo y movimiento, y la pedagogía afectiva en sí misma. En segundo lugar, generaremos unos métodos adaptados a las necesidades de la investigación que se adentran a una metodología de investigación basada en las artes (IBA) (Koro-Ljunberg, Suominen, Kallio-Tavin, Hernández-Hernández, VV.AA). Para continuar, se explorará cómo el marco teórico intracciona ${ }^{1}$ (Barad, 2007) con las sesiones de psicomotricidad de la Escuela Drassanes y el centro Tariqa. Finalmente, abriremos cuestionamientos sobre cómo transitan los afectos del movimiento en un evento localizado y qué nuevas aportaciones pueden generar en el campo de la pedagogía.

\section{Afectos}

El giro afectivo se ha consolidado como una perspectiva filosófica establecida, desde la epistemología feminista que data su origen en los años noventa, gracias a los trabajos de Brian Massumi y Gilles Deleuze, principalmente. Sin embargo, la genealogía de los mismos comienza mucho antes con la filosofía de Spinoza. Dependiendo de la interpretación que se ofrezca

1.- La intracción es un término de Barad (2007 en Arlander, 2014) que, a diferencia de la interacción, apuesta por relaciones donde los elementos se combinan para la acción, no como el resultado de ella (2014: 28, 30). Es decir, se prima una ontología relacional donde la unidad de análisis mínima es una relación y no elementos diferenciados en sí mismos. 
Hacia una pedagogía afectiva del movimiento / Towards an affective pedagogy of movement https://dx.doi.org/10.17561/rtc.n16.1

del concepto spinoziano, los afectos materializan la capacidad del cuerpo para transformarse (Hickey-Moody, 2016), huellas historiográficas de una política afectiva de los sentimientos (Bargetz, 2014), unidades empíricas radicales (Clough, 2009), entre otros. Sin embargo, lo que todas estas teorías tienen en común es la definición del mismo como momentos casi imperceptibles que, en la experiencia relacional con el mundo, posibilitan la modificación a uno mismo o una misma y a los y las demás.

El afecto, pues, circula por los cuerpos, tanto humanos como no humanos.

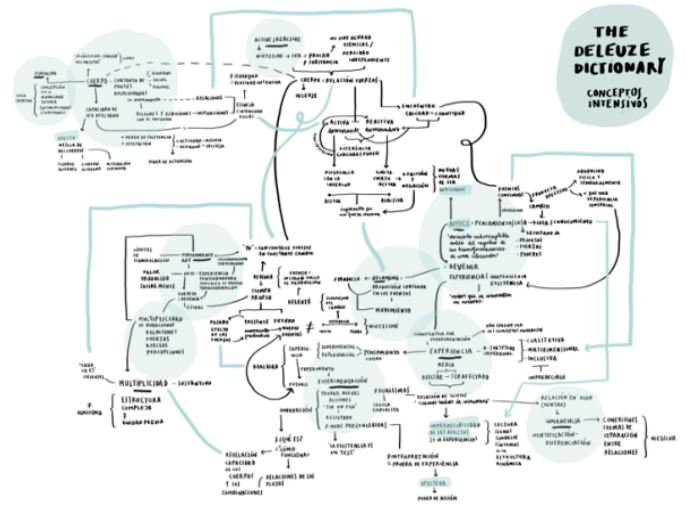

Figura 1: Cartografía de Parr (ed.) (2010) The Deleuze Dictionary Revised Edition. Edinburgh: Edinburgh University Press.

Cuando estos cuerpos se encuentran y se relacionan en un momento concreto, se ven afectados y experimentan modificaciones como consecuencia de su interacción y la relación con dicha experiencia (Hickey-Moody, 2016: 260). El afecto, pues, es una expresión de cambio (Colman, 2010: 12) que nos acerca a diversas y nuevas partes nuestras ya que la performatividad de la subjetividad está imbricada por la afectividad construyendo un 'yo' que se convierte en un catalizador para la materialización del pensamiento y las acciones (Colman, 2010: 544).

Entender el afecto, entonces, nos sitúa dentro de una lógica relacional donde la realidad es un flujo de fuerzas (Deleuze, 1992: 317 en Baugh, 2010: 94) en constante transformación. Este hecho nos aleja del giro epistemológico de las ontologías dualistas de la filosofía occidental dominante, donde el cuerpo es irracional y se desplaza en un lugar por debajo de la mente (Ahmed, 2013: 3). Desde esta perspectiva, los cuerpos existen cuando se ponen en relación y los afectos surgen de dicha circulación. 
Revista de Estudios en Sociedad, Artes y Gestión Cultural

www.terciocreciente.com http://revistaselectronicas.ujaen.es/index.php/RTC
Número 16

Julio 2019

Investigación
ISSN: 2340-9096

DOI: https://dx.doi.org/10.17561/rtc.

https://dx.doi.org/10.17561/rtc.n16.1

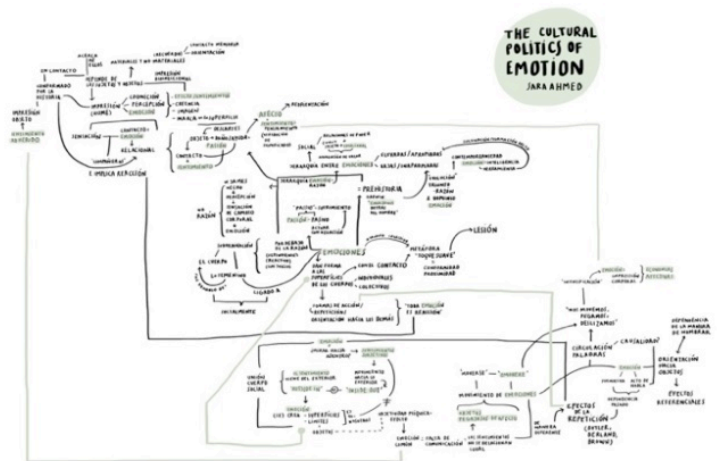

Figura 2: Cartografia de Ahmed, S. (2013) Introduction. Feel Your Way. En The Cultural Politics of Emotion (2a Ed.) (1-19). Abingdon. Oxon: RoutledgeRevised Edition. Edinburgh: Edinburgh University Press.

Al incorporar el afecto en la manera de ver el mundo nuestra concepción del cuerpo se transforma. Si el cuerpo siempre está en movimiento no tiene propiedades inherentes; es la relación de fuerzas entre los cuerpos humanos y no humanos la que permite definir los cuerpos en un momento concreto (Deleuze en Baugh, 2010). Por ende, los cuerpos son multiplicidades que "no hace[n] referencia a una unidad previa» (Roffe, 2010: 181). Se crean en la intracción: en una relación que permite la acción y no es el resultado de ella (Barad, 2007 en Arlander: 28, 30).

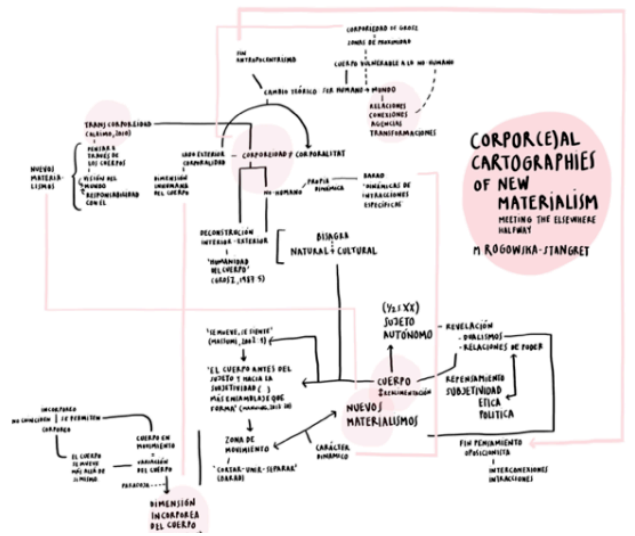

Figura 3: Cartografía de Rogowska-Stangret, M. (2017) Corpor(e)al Cartographies of New Materialism: Meeting the Elsewhere Halfway. En The 
Hacia una pedagogía afectiva del movimiento / Towards an affective pedagogy of movement https://dx.doi.org/10.17561/rtc.n16.1

Los cuerpos, entonces, se forman a partir del contacto que tienen con el mundo (Ahmed, 2013: 1). En este contacto los cuerpos se impresionan (ibíd: 6), se marcan por el afecto. Es esta impresión la que permite la transformación. Dicho de otro modo: el cuerpo se materializa durante el medio y no como materia diferenciada (Manning, 2013: 30 en Rogowska-Stangret, 2017: 59). Su poder no está determinado (Tiainen y Parikka, 2013: 209), se genera en la circulación de afectos. El cuerpo no contiene el afecto, pero su relación con el mundo le permite crearlos y recibirlos. Esta comprensión del cuerpo nos aleja de una visión antropocéntrica. El cuerpo se ve "afectado externamente» (Grosz, 1994 en Rogowska-Stangret, 2017: 62): está en una zona de proximidad con aquello que se relaciona.

¿Qué sucede, entonces, cuando entendemos que un cuerpo está en movimiento? Partiendo de la base de que la realidad fluye siempreal moverse, un cuerpo en movimiento es más afectivo:

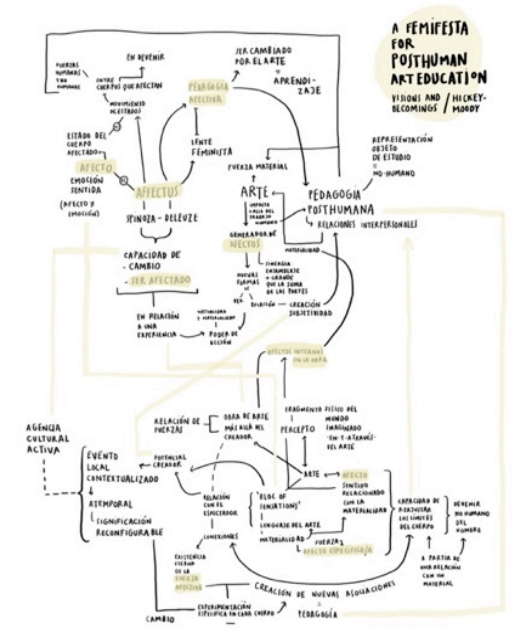

Figura 4: Cartografía de Hickey-Moody A. (2016) A Femifesta for Posthuman Art Education: Visions and Becomings. En Taylor C.A y Hughes C. (Eds.) Posthuman Research Practices in Education (258-

266.). London: Palgrave Macmillan.

«Cuando un cuerpo está en movimiento, no coincide con él mismo». Coincide con su propia transición: su propia variación. En movimiento, un cuerpo está en una relación inmediata y de desarrollo con su propio potencial no representado para variar. (Massumi, 2002: 4 en Rogowska-Stangret, 2017: 60)

Así pues, un cuerpo en movimiento varia, rompe sus límites y va hacia su potencial. Un cuerpo en movimiento intensifica las experiencias corporales (Ahmed, 2015: 56) ya que materializa la circulación de los afectos, visibiliza la fuerza del cambio y altera el estado del cuerpo (RevellesBenavente, 2017: 139). Esta alteración invita a los cuerpos a ver otras formas de entender y relacionarse (Hickey-Moody, 2016: 258) no con el objetivo de dar sentido al mundo, sino de explorar las posibilidades de devenir en el mundo (O’Sullivan, 2001: 130). 
Revista de Estudios en Sociedad, Artes y Gestión Cultural

www.terciocreciente.com http://revistaselectronicas.ujaen.es/index.php/RTC
Número 16

Julio 2019

Investigación
ISSN: 2340-9096

DOI: https://dx.doi.org/10.17561/rtc.

https://dx.doi.org/10.17561/rtc.n16.1

Anna Hickey-Moody (2016) describe un cierto paralelismo entre arte y la capacidad de transformación de los cuerpos:

El arte puede reajustar lo que una persona es o puede entender, producir y conectarse. Esto no quiere decir que una obra de arte cambie necesariamente a sus espectadores de formas prescritas, sino que las obras de arte pueden crear nuevas asociaciones. (2016: 261)

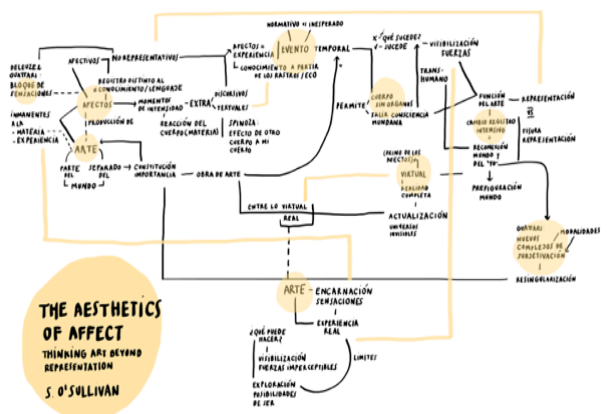

Figura 5: Cartografía de O'Sullivan, S. (2010) The aesthetics of affect: Thinking art beyond representation. En Angelaki: journal of theoretical humanities, 6(3), 125-135.

Desde la perspectiva de los afectos, el movimiento también proporciona la capacidad de cambio: los afectos surgidos en movimiento pueden separarse de sus orígenes temporales y geográficos y convertirse en entidades independientes (ibíd.) para modificar significados y relaciones. Esto hace posible que se pueda "pensar a través de los cuerpos» (Alaimo, 2010: 2 en Rogowska-Stangret, 2017: 64), a través de un movimiento que nos acerca a aquello que parece inaccesible a la conciencia (de Man en O'Sullivan, 2001: 126) y que solo se puede llegar a partir de la experiencia. Los afectos solo se pueden experimentar (O'Sullivan, 2001: 1), no son registrables. Sin embargo, sí que se puede ver su trazo, su eco (de Man en O’Sullivan, 2001: 126). Esto hace comprender el foco de la investigación: no es tan importante qué son los afectos, sino qué permiten hacer los afectos de un cuerpo en una situación pedagógica de movimiento.

\section{Hacia una pedagogía afectiva}

El cuerpo en movimiento tiene la capacidad de afectar y ser afectado. Es este tránsito de afectos encarnados el que permite la transformación hacia nuevas maneras de ver el mundo.

En consecuencia, una situación donde un cuerpo está en movimiento es potencialmente pedagógica. Esta potencialidad viene, por una parte, de la posibilidad que tiene el afecto de 
Hacia una pedagogía afectiva del movimiento / Towards an affective pedagogy of movement

https://dx.doi.org/10.17561/rtc.n16.1

«existir y de conservarse en sí mismo» (Deleuze y Guattari, 1996: 166 en Hickey-Moody, 2016: 261), aunque solo haya durado un instante. Es el poder de impresión el que nos permite entender que «[l]a persona que experimenta la fuerza producida por un afecto puede retener esta fuerza, y también puede cambiarse como resultado de su experiencia» (Deleuze y Guattari, 1996: 166 en Hickey-Moody, 2016: 261).

Por otra parte, la posibilidad de la persistencia del afecto es el detonante del potencial de aprender a partir de «escenas significativas» (Denzin, 1997: 207-208 en Hernández-Hernández, 2010: 16) generadas a partir de prácticas que cambian el registro del mundo (O’Sullivan, 2001: 128). No estamos hablando de concebir estrategias para generar afectos, ya que estos siempre están en circulación y son inevitables, sino prácticas que ayuden a aprender a conocerlos (Camps, 2011: 68); prácticas que tengan en cuenta que los afectos son singulares en cada cuerpo (ibíd: 74) y que, por lo tanto, es necesario atender a las especificidades de los flujos afectivos para buscar la potencialidad de cada individuo (ibíd: 73) y transformar no solo los cuerpos, sino la manera de entender el aprendizaje.

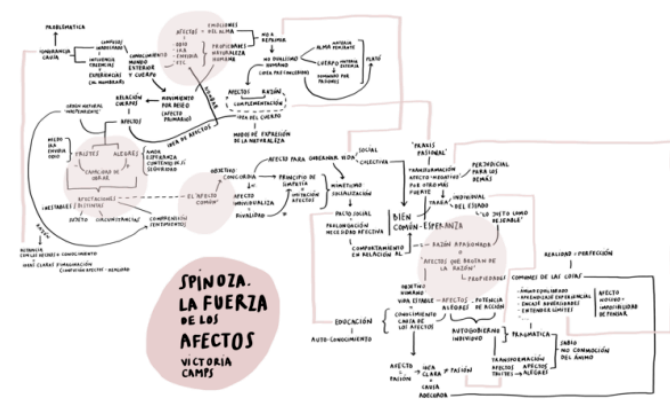

Figura 6: Cartografía de Camps, V. (2011). Spinoza. La fuerza de los afectos. En El gobierno de las emociones (65-86). Barcelona: Herder.

Sin embargo, atender los cuerpos es, desde nuestra perspectiva, sinónimo de transformar la pedagogía. Estamos apuntando la importancia de tomar en consideración la experiencia corpórea de aprender, la "materialidad del conocimiento" (Vidiella, 2012: 81). Estamos resaltando la importancia de explorar los cuerpos en «diferentes espacios físicos y subjetivos del aprendizaje» (ibíd: 91). Es en ellos donde se puede atender la circulación de afectos como instrumento de estimulación de un aprendizaje. De esta manera es posible que los cuerpos "colonizado[s] por el prejuicio del diagnóstico y la clasificación» (Hernández-Hernández, 2011: 18) puedan ser tomados en consideración desde una nueva mirada. Por un lado, si tomamos el cuerpo como devenir afectivo, siempre transformándose, parece que este se puede reinventar a partir de experiencias (Hernández-Hernández, 2010: 11), haciendo imposible su clasificación. Por el otro, si entendemos el cuerpo en movimiento como aquello que tiene la potencialidad de generar fuerzas que transforman y que alteran el significado del mundo (Revelles-Benavente, 2017: 141), ya no hay cuerpos que puedan ser encasillados. Los cuerpos en una pedagogía afectiva del movimiento, entonces, no son: devienen. 
Revista de Estudios en Sociedad, Artes y Gestión Cultural

www.terciocreciente.com http://revistaselectronicas.ujaen.es/index.php/RTC
Número 16

Julio 2019

Investigación
ISSN: 2340-9096

DOI: https://dx.doi.org/10.17561/rtc.

https://dx.doi.org/10.17561/rtc.n16.1

Ir hacia una pedagogía afectiva del movimiento nos adentra en cuestiones que repiensan las prácticas pedagógicas hegemónicas. Nos hace «habitar las normas de manera diferente» (Ahmed, 2015: 155) para ir más allá de los dualismos y las jerarquías, indagando las especificidades del cuerpo. Nos hace entender los cuerpos como participantes activos del aprendizaje, con el fin de adentrarnos en las relaciones (humanas y no humanas) que afectan y permiten transformar el propio sentido y el del mundo. Permite, pues, seguir desplazándonos para comprender la pedagogía desde otra mirada.

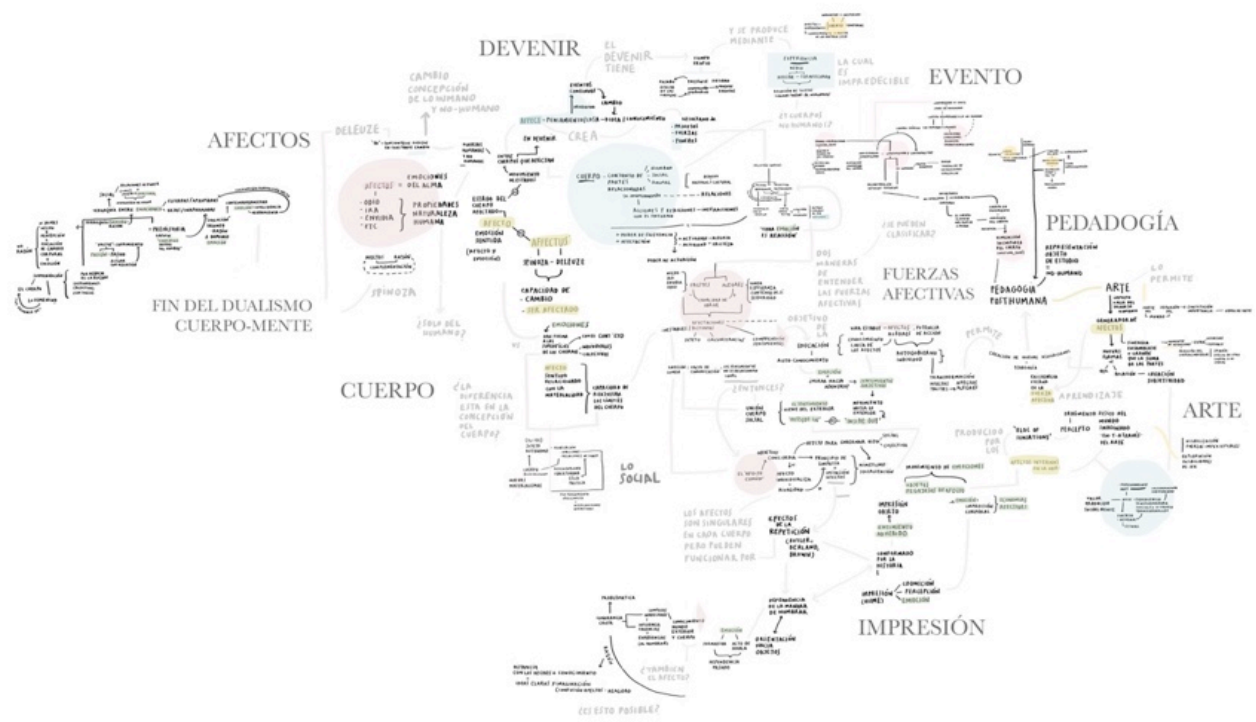

Figura 7: Cartografía de investigación donde se relacionan las cartografías de los distintos autores con el fin de elaborar el marco teórico.

\section{Objetivo}

El objetivo de este artículo es indagar en el significado de una pedagogía afectiva del movimiento y cuáles son las posibilidades que nos brinda esta línea. Esto equivale a explorar el sentido pedagógico del movimiento desde una mirada de los afectos. Significa, pues, acercarse a situaciones pedagógicas concretas donde el movimiento tiene lugar para que, a partir de los procesos de investigación, nos podamos hacer preguntas nuevas sobre cómo el cuerpo en movimiento es partícipe del aprendizaje, qué papel tienen los afectos y qué potencial tiene abrir esta mirada de investigación. 
Hacia una pedagogía afectiva del movimiento / Towards an affective pedagogy of movement

https://dx.doi.org/10.17561/rtc.n16.1

Para llevar a cabo este estudio, nos acercamos a las sesiones de psicomotricidad de la Escuela Drassanes y el centro Tariqa a partir de la IBA (Koro-Ljunberg, Suominen, KallioTavin, Hernández-Hernández, VV.AA). De esta manera es posible centrarnos en los procesos donde los afectos circulan por los cuerpos de los niños y las niñas en una situación pedagógica de movimiento determinada. Solo así dejamos de lado la voluntad de simplificar la realidad para mostrar nuestra intención de abrir nuevas discusiones que nos planteen qué aporta la perspectiva nuevo materialista al explicitar los conceptos de afecto y cuerpo, dentro del campo de la educación. Debates que nos lleven, pues, hacia una pedagogía afectiva del movimiento.

\section{Metodología}

La metodología de este artículo se basa en una IBA (Koro-Ljunberg, Suominen, Kallio-Tavin, Hernández-Hernández, VV.AA) ya que permite acercarse a los objetivos del artículo debido a que se parte de la atención a los procesos y a «los flujos afectivos de los elementos en la investigación» (Fox y Alldred, 2015: 411).

Esta combinación nos lleva a usar herramientas artísticas para indagar las relaciones y «explorar caminos de la experiencia que los dispositivos numéricos y narrativos [han mantenido] invisibles» (VV.AA, 2017: 1). Para ir más allá del lenguaje como referencia de un mundo literal, usamos dos métodos reinventados en esta investigación, que son las cartografías de investigación y las cart(e)ografías. Estos métodos permiten materializar los afectos producidos en un encuentro pedagógico de movimiento (Deleuze, 188: 127 en Fox y Alldred, 2015: 402) para entender el papel del afecto y del cuerpo en una situación de movimiento concreta.

\section{Las cartografías de investigación}

Las cartografías de investigación (Ruitenberg, 2007) son dispositivos visuales que permiten desbloquear y movilizar relacionalmente conceptos para generar nuevas conexiones (Deleuze y Guattari, 1994 en Lenz, 2016: 213). Este método resulta útil a la hora demostrar el proceso de aproximación a los referentes como "una herramienta para la comprensión de los demás» (Suominen, Kallio-Tavin, Hernández-Hernández, 2017: 104).

Podemos afirmar que las cartografías documentan el proceso de investigación a partir de la materialización de las conexiones de quien realiza una investigación en el ámbito que nos ocupa, tal y como se ha podido comprobar durante este estudio gracias a las figuras que hemos proporcionado hasta ahora. Por otra, posibilitan la alteración de los significados de los conceptos en la relación entre textos. Este movimiento es un movimiento metodológico y ético ya que resalta el modo en que las genealogías y los marcos teóricos en los que nos movemos tienen su propio dinamismo y su propia vitalidad. Cuando los hacemos visibles materializamos la 
Revista de Estudios en Sociedad, Artes y Gestión Cultural

www.terciocreciente.com http://revistaselectronicas.ujaen.es/index.php/RTC
Número 16

Julio 2019

Investigación
ISSN: 2340-9096

DOI: https://dx.doi.org/10.17561/rtc.

https://dx.doi.org/10.17561/rtc.n16.1

vitalidad de la producción de conocimiento y cómo se va transformando a medida que avanza la investigación, igual que también ayudan a la investigadora o investigador a ordenar el conocimiento. Además, la teoría y la formación teórica, son en sí mismas una materialización de la política. En 1929, Virginia Wolf instaba a las mujeres a tener una habitación propia para poder así ejercer una agencialidad empoderadora, aunque se limitase a las paredes de esa habitación. Casi 100 años después, es importante situar las investigaciones que hacemos dentro de un "canon científico corporizado" (Revelles-Benavente, en revisión). Este canon representa la relación que hay entre las disciplinas teóricas y los investigadores e investigadoras, pero también compañeras, profesoras y activistas que nos ayudan dentro de estos procesos cognitivos. Situar el conocimiento es un gesto ético hacia el cuerpo de conocimiento que se ha creado antes y pueden ser tecnologías que ayuden a confrontar discursos socio-políticos hegemónicos.

\section{Las cart(e)ografías de los afectos}

Las cart(e)ografías son un método que permite aterrizar el marco teórico a partir de reconceptualizar el concepto de cartografía. Estas se diferencian del concepto anterior (aunque genealógicamente devienen del anterior) y surgen de la intracción entre coreografía y cartografía. Su práctica significa mapear los rastros del movimiento y del afecto una vez este ha sido generado en una sesión de psicomotricidad. Se pueden denominar, pues, cart(e)ografías de los afectos, en tanto que detienen en un momento concreto los tránsitos afectivos, aunque solo hayan durado un instante, y los materializan a partir del arte para poder transformarse.

Esta práctica se planteó a los distintos grupos que conforman las clases de Educación Primaria:

- Las cart(e)ografías se realizaron en un grupo de primero de primaria (6 años) del centro Drassanes, donde el alumnado se encuentra en una edad psicomotora. Drassanes permite ver cómo se mueven los afectos en una sesión de psicomotricidad en un grupo dentro de la escuela ordinaria. Sesiones donde, durante hora y media, se experimenta el movimiento entre la totalidad del alumnado (19) y sus tres maestras con un fin pedagógico.

- Se plantearon las cart(e)ografías en dos sesiones del centro Tariqa: uno con niños (solamente de género masculino) de entre 7 y 10 años; y otro con niños de 10 a 13 años, donde se supone que la relación con el cuerpo y el movimiento ya está consolidada. En las dos sesiones de Tariqa, con la misma duración que en Drassanes, los grupos son reducidos, de entre 3 y 4 niños. Estos son acompañados/as por 2 o 3 docentes que se reúnen en el centro después del horario lectivo para desarrollar la psicomotricidad como estrategia de atención a sus problemáticas de aprendizaje.

Estos tres eventos de movimiento permiten ver diferentes flujos afectivos producidos por las distintas situaciones: número y edad del alumnado, número de docentes y objetivos sobre 
Hacia una pedagogía afectiva del movimiento / Towards an affective pedagogy of movement

https://dx.doi.org/10.17561/rtc.n16.1

los que se cimenta la sesión. El método se usa de la misma forma en los tres casos: dejando realizar la sesión de una hora de psicomotricidad por el/la educador/a y, después, proponiendo la práctica cart(e)ográfica (durante 30 minutos) para vislumbrar los afectos y aquello sucedido durante el movimiento. Para ello, se propone primero que se marque el movimiento ejecutado en lápiz de grafito. En un segundo momento, se propone trazar con lápices de colores los momentos afectivos.

El análisis de las cart(e)ografías se centra en las relaciones afectivas ocurridas en su generación dentro de diferentes contextos, pero bajo unas mismas premisas. No se busca interpretar las cart(e)ografías como una realidad con un único significado, sino que se revisan los procesos de creación que, parciales y temporales, permiten un conocimiento singular sobre la interpretación del mundo (Souminen, Kallio-Tavin y Hernández-Hernández, 2017: 107) y el tránsito de los afectos en una situación pedagógica de movimiento localizada.

\section{Resultados}

Como se ha apuntado en la sección anterior, en el trabajo de campo se atiende al proceso de generación de las cart(e)ografías y no a la sesión de psicomotricidad en sí. De esta manera es posible atender los afectos retenidos en el movimiento a partir de la generación de cart(e) ografías.

\section{Las cart(e)ografías en Drassanes}

En Drassanes, el método se propuso el día 9 de mayo de 2018. En la generación de cart(e) ografías los alumnos iniciaron su práctica sin cuestionarla. Su proceso parecía no previsto, mostrando cómo su cuerpo era capaz de recordar sus movimientos y afectos para materializarlos en el papel. El lápiz se movía en intracción con un cuerpo que podía regenerar sus tránsitos y mostraba la actividad de la materia como un participante activo e indispensable (Kontturi, 2013: 18) e igual creador de estas cart(e)ografías. Los afectos, pues, estaban circulando por los cuerpos tanto humanos como no humanos para generar un nuevo dispositivo.

Este nuevo dispositivo, la cart(e)ografía, albergaba más que las palabras del alumnado. Esta afirmación viene dada por aquello percibido durante la sesión: la dificultad por verbalizar el movimiento y las afectaciones. Sin embargo, trazar los momentos afectivos no supuso ningún conflicto. Se ponía en relieve la posibilidad de ver el eco de los afectos una vez experimentados (de Man en O'Sullivan, 2001: 126) y, en consecuencia, la necesidad de acceder a ellos de otra forma más allá del lenguaje literal.

Al utilizar métodos artísticos, se pudo acceder al origen de los momentos afectivos. Las impresiones marcadas en el papel tenían que ver con las relaciones ocurridas en el movimiento 
Revista de Estudios en Sociedad, Artes y Gestión Cultural

www.terciocreciente.com http://revistaselectronicas.ujaen.es/index.php/RTC
Número 16

Julio 2019

Investigación
ISSN: 2340-9096

DOI: https://dx.doi.org/10.17561/rtc.

https://dx.doi.org/10.17561/rtc.n16.1 de la hora de psicomotricidad: algunos niños relacionaron momentos de intensidad con retos físicos, saltar más alto, no poder saltar, marcar un gol, bajar por un tobogán o, incluso, hacerse daño. Los momentos afectivos de las niñas fueron construir una casa, jugar en grupo o la imposibilidad de acercarse a un colectivo, entre otros.

En ambos casos sus afectos tenían que ver con encuentros relacionales con cuerpos humanos y no humanos que les permitía descubrirse como cuerpo. Estos momentos de intensidad eran dados por una relación de fuerzas materiales que les permitía explorar posibilidades (en cuestión de potencialidades corporales o bien de reajustar los cuerpos en las construcciones sociales de grupo) para poder reconfigurarse. Un reajuste que hace visible la posibilidad de intensificar las experiencias corporales a partir de la relación con el mundo (Ahmed, 2015: 56). Estas experiencias son las que permiten verse afectado y afectar cuando se consigue experimentar los límites: para dar cuenta de la materialidad de su cuerpo, de los límites de las relaciones y de la construcción social de los grupos. Unos límites que permiten transformar el sentido del 'yo' y del mundo.
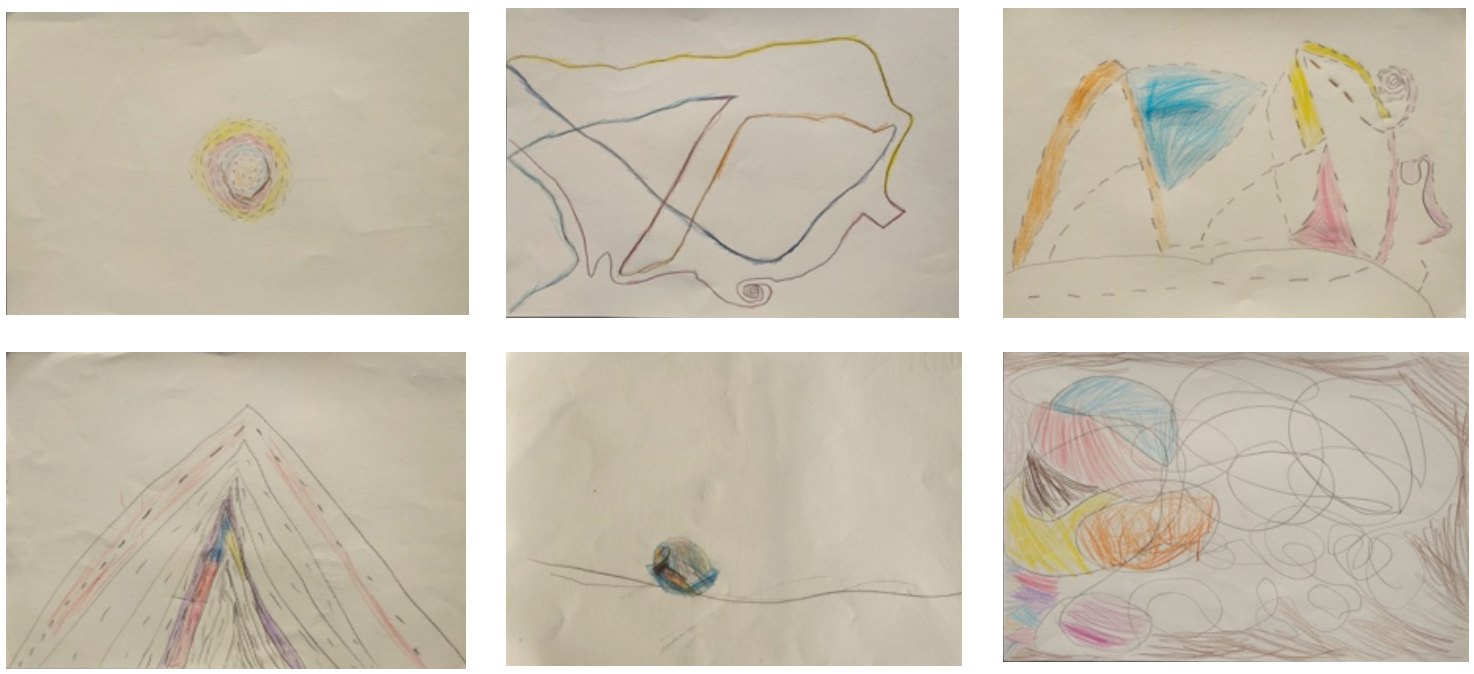

Figura 8: Cart(e)ografías realizadas por el alumnado de primero de primaria de la Escuela Drassanes el día 9 de mayo de 2018. 
Hacia una pedagogía afectiva del movimiento / Towards an affective pedagogy of movement https://dx.doi.org/10.17561/rtc.n16.1

Así pues, en la generación de las cart(e)ografías en Drassanes se puede ver que estas albergan la interpretación de los recorridos y momentos de afecto del alumnado a partir del lenguaje plástico. Los trazos materializan el movimiento según la peculiaridad de cada cuerpo, permitiendo ver sus variaciones, temporalidades, intracciones (Tiainen y Parikka, 2013: 208-209), su forma de ver el mundo más allá de la consciencia mundana (Baudiou, 1984: 5 en O'Sullivan, 2001: 127). En el trazo de los afectos se puede ver como estos se interpretan en sus diferentes formas según las circunstancias de cada cuerpo (Camps, 2011: 70). Igualmente, cabe resaltar la posibilidad de los afectos de retenerse y conservarse en sí mismos para materializarse a partir del arte y crear nuevas imágenes entorno a lo vivido (Hickey-Moody, 2016: 261).

\section{Las cart(e)ografías en Tariqa}

El jueves 17 de mayo de 2018 se elaboraron las cart(e)ografías en los dos grupos de Tariqa. Al atender a niños con dificultades de relación y aprendizaje se pudo reafirmar cómo la especificidad de cada cuerpo afectado en movimiento se materializaba sobre el papel.

A diferencia del alumnado que participó en Drassanes, los dos grupos de Tariqa eran de entre 3 y 4 niños que acudían al centro para trabajar, a partir de la psicomotricidad, problemáticas relacionadas con el aprendizaje y las relaciones ${ }^{2}$.

2,- Iolanda Vives, directora del centro Tariqa Psicomotriu, concedió una entrevista para poder compartir las bases de su trabajo y facilitar el desarrollo de la investigación. 
Revista de Estudios en Sociedad, Artes y Gestión Cultural

www.terciocreciente.com http://revistaselectronicas.ujaen.es/index.php/RTC
Número 16

Julio 2019

Investigación
ISSN: 2340-9096

DOI: https://dx.doi.org/10.17561/rtc.

https://dx.doi.org/10.17561/rtc.n16.1
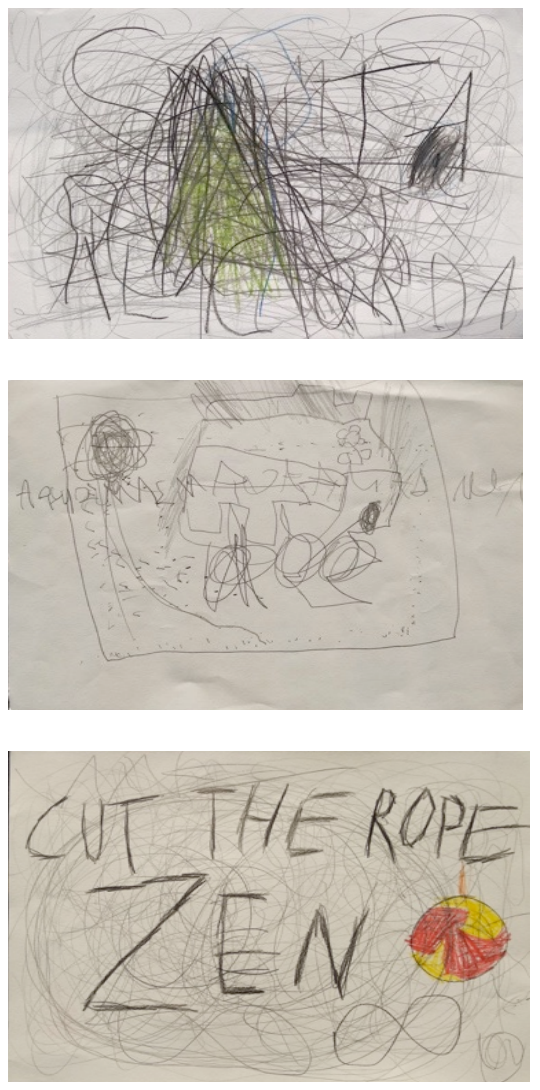

Figura 9: Cart(e)ografías realizadas por los niños del primer grupo del centro Tariqa el día 17 de mayo de 2018.
El primer grupo, de niños de entre 7 y 10 años, mostró ilusión en experimentar la realización de las cart(e)ografías. Sin embargo, sus cuerpos no frenaban el movimiento. Esto repercutió en que su trazo mostrara una intensidad imparable que se alejaba de explorar el movimiento y los afectos de la sesión anterior. Los cuerpos seguían en una afectación que los estimulaba de manera que siempre seguían en variación continua. Esto puede dar pistas sobre su dificultad de relación y aprendizaje: al estar en constante movimiento de intensidad se imposibilita explorar los afectos retenidos.

El caso del segundo grupo, de niños entre $10 \mathrm{y}$ 13 años, mostró resistencias para hacer las cart(e) ografías. Los niños se negaron a indagar en su movimiento y afectos de la sesión psicomotriz. Parecía que no había la posibilidad de acceder a sus impresiones, aunque estas hubieran existido por la naturaleza del afecto de estar en constante circulación. Por lo contrario, cada uno se adentró en diversos estados huyendo de la propuesta. Uno se sumergió en un movimiento imparable, otro se alejó de la propuesta generando un mapa de datos sobre las posibilidades de peligro que tiene el movimiento. El tercero hizo un mapa relacionado con un videojuego, ya que en movimiento se había sentido como si estuviera allí. Mostraba el potencial de su cuerpo en movimiento para ir más allá del mundo tangible (Manning 2007: 136 en Tiainen y Parikka, 2013: 222). El cuarto realizó un mapa para medir el afecto. 
Hacia una pedagogía afectiva del movimiento / Towards an affective pedagogy of movement

https://dx.doi.org/10.17561/rtc.n16.1
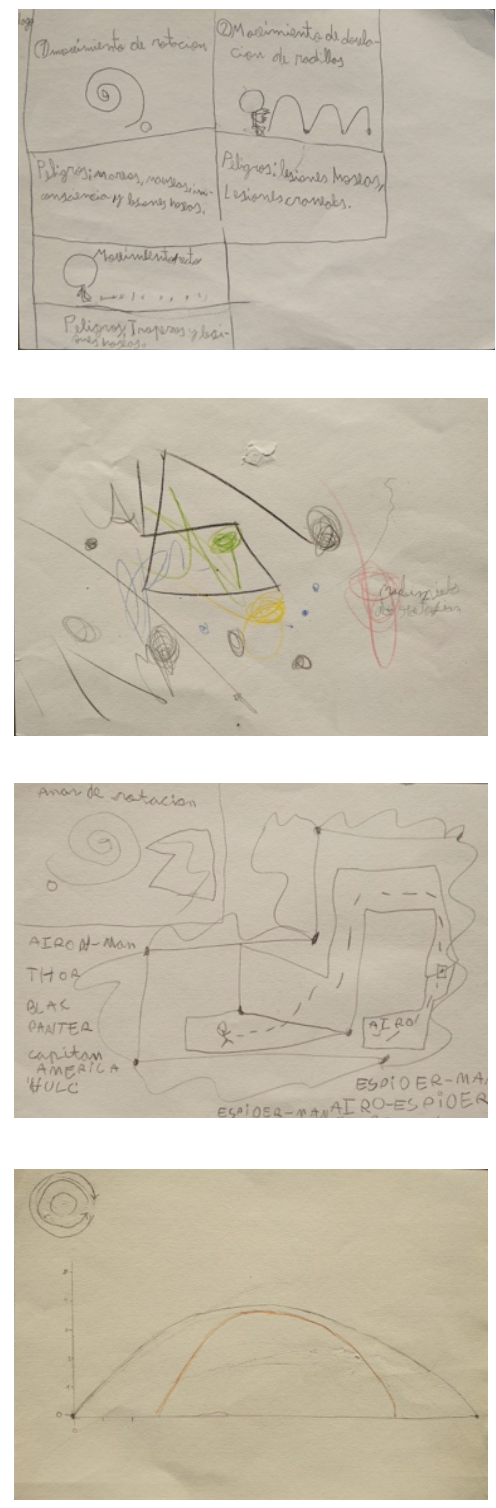

Figura 10: Cart(e)ografías realizadas por los niños del segundo grupo del centro Tariqa el día 17 de mayo de 2018.
Después de realizar la propuesta con los distintos grupos, se pudo afirmar que el uso de estrategias pedagógicas como las cart(e)ografías permite acercarse a los afectos y visibilizarlos, constatado en el caso de Drassanes. Por esta razón, es necesario que en casos de dificultad de relación y aprendizaje se pueda disponer de más tiempo y repetir con más frecuencia prácticas que acerquen a los momentos afectivos. De esta manera, es posible romper las barreras entre el investigador o investigadora y alumnado con dificultades de relación y aprendizaje y brindar la posibilidad de indagar en grupos como los de Tariqa, no para diagnosticar una problemática que estigmatice al alumnado, más bien al contrario, para poder aproximarse a las afectaciones ocurridas en relación y en movimiento con el objetivo de que ellos puedan empezar a reconocerlas. De esta manera es posible permitir al alumnado acceder al conocimiento de su cuerpo y su potencial para mejorar su relación con el mundo, las relaciones y el aprendizaje.

\section{Discusión}

En el presente artículo hemos elaborado un marco teórico basado en los nuevos materialismos con el objetivo de explorar el sentido pedagógico del movimiento desde el afecto. En un segundo lugar, hemos usado una metodología de IBA que se divide en dos métodos reconfigurados bajo esta investigación. Por último, hemos tejido hilos entre aquel conocimiento surgido del marco teórico con una experiencia situada de movimiento en un entorno pedagógico, en concreto, bajo 3 sesiones de práctica psicomotriz en distintos grupos y lugares. En la investigación, se atiende a los procesos para poder abrir nuevos cuestionamientos. Por esta razón, a continuación, iniciamos nuevos caminos partiendo de la reflexión que se genera desde la elaboración de las cart(e)ografías: 


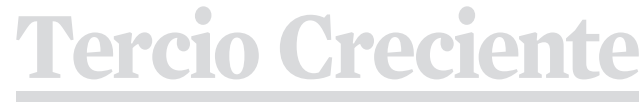

ISSN: 2340-9096

DOI: https://dx.doi.org/10.17561/rtc.
Revista de Estudios en Sociedad, Artes y Gestión Cultural

www.terciocreciente.com http://revistaselectronicas.ujaen.es/index.php/RTC
Número 16

Julio 2019

Investigación

https://dx.doi.org/10.17561/rtc.n16.1

1) Por una parte, extraemos la necesidad de seguir explorando experimentalmente y fluyendo por flujo (Baugh, 2010: 95) con métodos que revelan modos de hacer que van más allá del pensamiento lineal, que se sumergen en las multiplicidades para romper lo que las palabras pueden limitar. Metodos que atiendan a los procesos más que a los resultados para transferir nuevos conocimientos. En el caso de este artículo, los métodos usados son útiles para seguir pensando desde otras praxis diversas, como las artes. Al fin y al cabo, tanto las cartografías de investigación como las cart(e)ografías son dispositivos visuales que tienen un mismo fin: plasmar los movimientos de quien los realiza no de manera fija, sino posibilitando que, de aquel nuevo movimiento de ejecución, se pueda ir más allá y construir nuevos relatos que tienen que ver con aprendizajes donde el cuerpo tiene lugar. En el caso de las cartografías de investigación, permiten materializar conceptos abstractos en un momento concreto para dialogar con los autores referentes en la investigación a partir de los flujos afectivos. Asímismo, las cart(e)ografías permiten materializar el movimiento vivido y los momentos de intensidad en un dispositivo para poder seguir avanzando en el devenir. Ambos tienen un mismo fin: permitir que el mapa retenga por un momento las conexiones de pensamientos y los movimientos corporales para desplazarse hacia nuevos movimientos que transforman y, por ende, son pedagógicos.

2) Haciendo hincapié en el método de las cart(e)ografias, afirmamos que estos dispositivos tienen dos líneas funcionales:

a) Las cart(e)ografías obran como un método que permite ver lo que un cuerpo puede hacer en una situación localizada de aprendizaje (Deleuze, 1988c: 125 en Baugh, 2010: 94). En el caso de esta investigación, materializan los afectos de un cuerpo en movimiento, como se ha podido ver en Drassanes. En el caso de Tariqa, posibilitan ver la dificultad para atender los tránsitos afectivos en una situación pedagógica de movimiento. En ambos casos, este dispositivo produce algo nuevo en sí mismo (Kontturi, 2013: 20) y permite aterrizar conceptos teóricos como el afecto y el cuerpo para dialogar con situaciones concretas de aprendizaje. Respaldándonos en los casos de estudio, vemos que las cart(e)ografías pueden ser el eco de un cuerpo en movimiento que va más allá la conciencia mundana (Baudiou, 1984: 5 en O’Sullivan, 2001: 127). Esto nos permite dialogar con las posibilidades que tiene el arte y el movimiento para materializar afectos. Sin embargo, la importancia no se halla en el mero hecho de materializar, sino en aquello que se abre al hacerlo. Con esto nos referimos a la oportunidad que brindan las cart(e)ografías de acceder a aquello a lo que las palabras no llegan: son las especificidades de cada cuerpo y su interpretación del mundo a partir de los propios afectos de niños y niñas. En el caso del alumnado de Drassanes se puede ver claramente: sus cart(e)ografías muestran un nuevo mundo visual en infinitud de formas construido a partir de los afectos retenidos en movimiento. Estos dispositivos, pues, permiten acercarnos a las transformaciones del alumnado, dialogar con sus relaciones y con aquello que les posibilita abrir nuevas miradas. Lejos de ser un instrumento de observación que se puede interpretar como una realidad medible u objetiva, las cart(e)ografías son un método que permite adentrarnos al mundo de 
Hacia una pedagogía afectiva del movimiento / Towards an affective pedagogy of movement

https://dx.doi.org/10.17561/rtc.n16.1

los afectos del movimiento y, por lo tanto, a las transformaciones y aprendizajes en devenir de los niños y las niñas.

b) Las cart(e)ografías son una estrategia pedagógica por la posibilidad que ofrecen de acceder a los afectos de quien las realiza y reconfigurar el evento de movimiento (Barad, 2007: 140 en Arlander, 2014: 28). Al reconfigurarlo, el movimiento se convierte en un movimiento diferente (Massumi, 2002:40-41 en Tiainen y Parikka, 201: 213) que permite traducir las afectaciones en un dispositivo material que va hacia nuevos tránsitos y habla de lo que ha afectado y transformado al alumnado; es decir, de lo que han aprendido y que siguen explorando. En sus líneas discontinuas, trazos circulares, intensidades compactas y universos dispersos niños y niñas pueden ir más allá de las delimitaciones de las palabras. En el caso de Drassanes, permiten recoger aquellos momentos relacionales con el resto de compañeros y compañeras y/o elementos materiales. Momentos de retos físicos o sociales que les posibilitan explorarse, reconocer su cuerpo dentro del aprendizaje y, al mismo tiempo, ver qué les afecta y reflexionar sobre su relación con el mundo. En el caso de los niños de Tariqa, las cart(e)ografías permiten ver las disonancias entre aquello vivido y aquello trazado, generando algo totalmente nuevo. Dan la posibilidad de entrever la dificultad de relación que en Drassanes no se percibió y el potencial pedagógico para buscar formas de acceder a sus afectos.

En ambos casos, las cart(e)ografías muestran la materialización de los afectos del movimiento y, de igual modo, el potencial pedagógico del cuerpo para convertirse: "relacionar, sentir, percibir y pensar de otra manera» (Tiainen y Parikka, 2013: 223). Sin embargo, esta materialización ocurre en momentos diferentes en los dos centros estudiados. En Drassanes se produce en la relación con la sesión de movimiento anterior; mientras que en Tariqa son los afectos que circulan en el momento de la realización del dispositivo. En los dos casos, se ve posible retener el momento de transformación donde el cuerpo se vuelve diferente al que era (ibíd: 213) y se explora y materializa a partir de las cart(e)ografías. Entonces, se puede ver cómo el cuerpo es partícipe y reconocida parte del aprendizaje. Los saltos, las relaciones sociales, el hacerse daño, todo aquello que se queda en forma de marca tiene, al fin, su lugar e importancia. Entonces, es a partir de materializar las conexiones afectivas (RevellesBenavente, 2017: 142) que se puede convertir el movimiento y sus afectos en nuevas formas de ver y relacionarse (Hickey-Moody, 2016: 251). Aquí reside el potencial pedagógico de las cart(e)ografías: la transformación de experiencias corporales como la construcción de una casa (en uno de los casos de Drassanes) en un material visual que no traduce figurativamente aquel momento de intensidad, sino que se reconfigura para revisar y extender los afectos y transformaciones. En esta revisión y toma de conciencia es en la que el niño o la niña puede seguir desplazándose hacia su potencial. En el caso de Tariqa, también se puede ver las alteraciones que proponen las cart(e) 


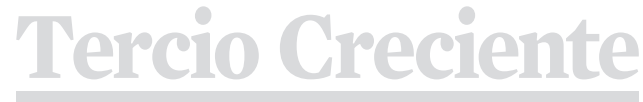

ISSN: 2340-9096

DOI: https://dx.doi.org/10.17561/rtc.
Revista de Estudios en Sociedad, Artes y Gestión Cultural

www.terciocreciente.com http://revistaselectronicas.ujaen.es/index.php/RTC
Número 16

Julio 2019

Investigación

https://dx.doi.org/10.17561/rtc.n16.1

ografías. Uno de los niños pasó del movimiento imparable a generar un mapa de datos sobre los peligros que tiene el movimiento. Esta muestra nos hace ver que consiguió llegar a otros planos de reflexión que, pese a estar inconexos con la sesión de psicomotricidad, nos revelan una transformación que va más allá de las palabras de aquel niño. Por esta razón, se puede afirmar que los afectos encarnados en la materia permiten transitar hacia pensamientos que se van de su origen temporal, que rompen con aquello preestablecido. Las cart(e)ografíaspueden establecer conexiones dentro de los mundos heterogéneos del alumnado en las sesiones de movimiento, trazando relaciones para negociar su ser (Schulte, 2016: 142) y su manera de aprender.

3) Por otra parte, la realización de cart(e)ografías como método y, a la vez, estrategia pedagógica permite acercarnos a una pedagogía afectiva del movimiento. Una pedagogía que encarna los afectos del movimiento e impresiona para producir cambios. Que permite retener momentos afectivos y explorarlos a partir de escenas significativas como la elaboración de unas cart(e)ografías que reconfiguran el propio cuerpo, aquello que afecta y la relación con el mundo. Una pedagogía que invita a alejarnos de las prácticas normativas en educación para explorar nuevos modos de hacer y pensar:

a) la pedagogía afectiva permite que los cuerpos, humanos y no humanos, de niños y niñas y de educadores, sean parte del aprendizaje. El movimiento del cuerpo, tanto en la sesión de psicomotricidad como después en la realización de cart(e) ografías, se vuelve parte indispensable de las afectaciones producidas y, a su vez, de las transformaciones que permiten el aprendizaje. En estos eventos, los cuerpos cogen presencia para ponerse en relación e intraccionar como manera de conocerse y volverse a conocer, rompiendo los límites del aprendizaje. Los cuerpos en una pedagogía afectiva, pues, exploran sus espacios físicos del aprendizaje y permiten ver la "materialidad del conocimiento» (Vidiella, 2012: 81), deshaciendo la dualidad entre cuerpo y mente aceptada por la mayor parte de la filosofía dominante.

b) la pedagogía afectiva posibilita atender las especificidades de los cuerpos. En la elaboración de cart(e)ografías hemos podido ver la medida en que cada cuerpo difiere de temporalidad según sus particularidades. Esto nos lleva a pensar en la importancia de generar eventos significativos (Denzin, 1997: 207-208 en Hernández-Hernández, 2010: 16) que atiendan aquellos que a los que son diagnosticados problemáticas de aprendizaje. Observamos, de manera clara en el caso de Tariqa, que las cart(e)ografías permiten acercarnos a aquellos cuerpos que parecen estar en un eterno movimiento, siempre estimulados, dando la impresión de que no pueden frenar por un momento y revisar sus flujos afectivos generados en movimiento. Esto nos puede revelar algunas de las problemáticas de relación y aprendizaje, no para clasificar, sino para ver qué nuevos caminos pueden propiciar otro tipo de aprendizaje que tenga en cuenta su especificidad y posibilite salir del estigma. Si acceder a los afectos permite indagar en 
Hacia una pedagogía afectiva del movimiento / Towards an affective pedagogy of movement

https://dx.doi.org/10.17561/rtc.n16.1

las transformaciones del alumnado, atender aquellos y aquellas que tienen dificultades para hacerlo puede proporcionarles acercarse a aquello que les afecta, a su propio cuerpo en relación con el mundo y, por lo tanto, a su potencial. Si en la generación de cart(e) ografías en Drassanes se pudo entender cómo los momentos afectivos tienen que ver con las intracciones con los cuerpos humanos y no humanos, propiciar estrategias pedagógicas que permitan visibilizar los afectos y trabajar desde ellos puede ayudar a aquellos con dificultades de aprendizaje a tener mejor relación con el mundo y, a consecuencia, con el aprender.

c) la pedagogía afectiva propone prácticas que afectan y que transforman. Buscan que niños y niñas exploren maneras de acceder a sus afectos con el objetivo de repensar su experiencia de aprendizaje e ir más allá. Prácticas que reconfiguran sus afectos a partir de expresiones materiales, como las cart(e)ografías, que no frenan el movimiento, sino que reproducen su potencial de transformación. Son estas propuestas las que permiten a los niños y a las niñas entenderse como seres en movimiento, siempre en relación, siempre en devenir. Son prácticas, pues, que les permiten reajustarse y transformarse según los eventos, encontrando nuevas formas de devenir y de aprender.

Este artículo invita a seguir explorando métodos y/o estrategias pedagógicas que atiendan a los afectos y permitan ir hacia una pedagogía afectiva. Métodos y/o estrategias que puedan ir más allá de la pedagogía normativa para empezar a utilizar métodos que recojan los momentos afectivos del movimiento y visibilicen las transformaciones que los niños y las niñas han vivido para permitir el devenir. Si lo importante es qué pueden hacer los afectos de un cuerpo en movimiento, parece que este enfoque abre posibilidades para atender esta cuestión. No simplificándola, sino generando nuevos relatos que, aun y específicos, puedan ser significativos para ver la relación con el mundo de manera nueva (Davies, 2014 en Schulte, 2016: 148). Construyendo, a partir de la IBA y los nuevos materialismos, maneras de atender a realidades subjetivas que, más allá del lenguaje, puedan acercarse a los afectos para proporcionar un aprendizaje de ser transformándose constantemente a partir de su relación con el mundo. Pequeños pasos hacia una pedagogía afectiva del movimiento donde los flujos afectivos del movimiento son la guía del devenir de un aprendizaje transformador. Un aprendizaje que se genera en el proceso, no buscando resultados finales. Un aprendizaje que aparta las jerarquías para entender las relaciones con aquello humano y no humano como motor de cambio. Una pedagogía que entiende el afecto como aquello indispensable para la transformación de los cuerpos en las relaciones pedagógicas. Una pedagogía que concibe los cuerpos en movimiento, nunca estables e igual de partícipes en el aprender. Una pedagogía afectiva que entiende el mundo y el aprendizaje como un movimiento tanto teórico como práctico para empezar a ver la pedagogía desde los desplazamientos. Una pedagogía afectiva del movimiento, pues, que nos puede ayudar a repensar cómo aprendemos para indagar en estrategias pedagógicas que afecten y transformen. En definitiva, para aportar, poco a poco, nuevas maneras de hacer en educación. 


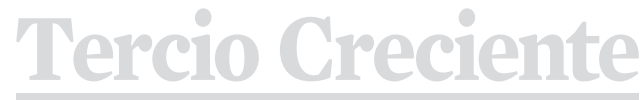

ISSN: 2340-9096

DOI: https://dx.doi.org/10.17561/rtc.

https://dx.doi.org/10.17561/rtc.n16.1
Revista de Estudios en Sociedad, Artes y Gestión Cultural

Número 16

Julio 2019

com

http://revistaselectronicas.ujaen.es/index.php/RTC

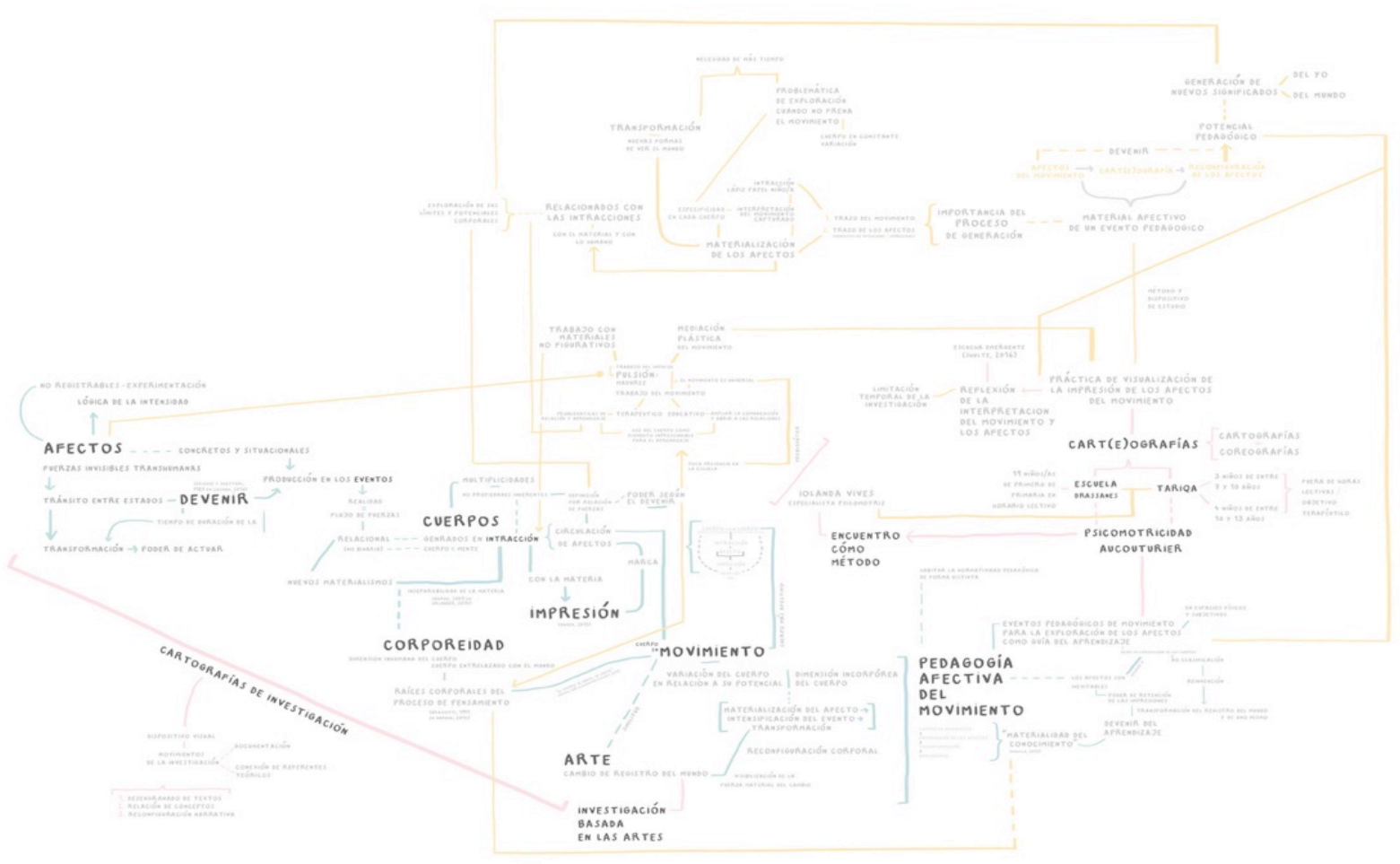

Figura 11: Cartografía resumen de la investigación. 
Hacia una pedagogía afectiva del movimiento / Towards an affective pedagogy of movement https://dx.doi.org/10.17561/rtc.n16.1

\section{Referencias}

Ahmed, S. (2013) Introduction. Feel Your Way. En The Cultural Politics of Emotion (2a Ed.) (119). Abingdon. Oxon: Routledge.

Ahmed, S. (2015) La contingencia del dolor. En La política cultural de las emociones (47-76). Ciudad de México: Universidad Nacional Autónoma de México.

Arlander, A. (2014) From interaction to intra-action in performing landscape. En RevellesBenavente, B., González Ramos A. Y Nardini K. (Coord.) New feminist materialism: engendering an ethic-onto-epistemological methodology. Artnodes, 14, 26-34. Recuperado el <15 de abril de 2018> de <http://journals.uoc.edu/ojs/index.php/ artnodes/article/view/n14-arlander/n14-arlander-en>

Baugh, B. (2010) Body. En Parr, A. (Ed.) The Deleuze Dictionary Revised Edition (35-37). Edinburgh: Edinburgh University Press.

Baugh, B. (2010) Experimentation. En Parr, A. (Ed.) The Deleuze Dictionary Revised Edition (93-95). Edinburgh: Edinburgh University Press.

Camps, V. (2011). Spinoza. La fuerza de los afectos. En El gobierno de las emociones (65-86). Barcelona: Herder.

CLOUGH, Patricia. 2009. The new empiricism. Affect and Sociological method, European Journal of Social Theory 12 (1): $43-61$

Colman, F. (2010) Affect. En Parr, A. (Ed.) The Deleuze Dictionary Revised Edition (11-13). Edinburgh: Edinburgh University Press.

Fox, N y Alldred, P. (2015) New materialist social inquiry: designs, methods and the researchassemblage. En International Journal of Social Research Methodology, 18:4, 399-414.

Hernández-Hernández, F. (2011) Pensar la relación pedagógica en la universidad desde el encuentro entre sujetos, deseos y saberes. Barcelona: Universitat de Barcelona. Dipòsit Digital. Recuperado el <15 de abril de 2018> de <http://hdl.handle.net/2445/20946>

Hernández-Hernández, F. (2010) Narrativas en torno a las experiencias de subjetividad en la escuela primaria. En Hernández-Hernández, F. (Coord.) Aprender a ser en la escuela primaria (9-25). Barcelona: Octaedro.

Hickey-Moody A. (2016) A Femifesta for Posthuman Art Education: Visions and Becomings. En Taylor C.A y Hughes C. (Eds.) Posthuman Research Practices in Education (258-266.). London: Palgrave Macmillan. 
Revista de Estudios en Sociedad, Artes y Gestión Cultural

www.terciocreciente.com http://revistaselectronicas.ujaen.es/index.php/RTC
Número 16

Julio 2019

Investigación

DOI: https://dx.doi.org/10.17561/rtc.

https://dx.doi.org/10.17561/rtc.n16.1

Kontturi, K.K. (2013) From double Navel to Particle-sign: Toward the a-signifying work of painting. En Barrett, E. y Bolt, B. (Eds.) Carnal Knowledge: Towards a 'New Materialism' through the Arts (17-27). Londres y Nueva York: I.B. Tauris.

Koro-Ljunberg, M. (2012) Researchers of the World, Create! En Qualitative Inquiry, 18, 808-818.

O’Sullivan, S. (2010) The aesthetics of affect: Thinking art beyond representation. En Angelaki: journal of theoretical humanities, 6(3), 125-135.

Revelles-Benavente, B. (2017) Affecting feminist pedagogies: performing critical thinking in between social networking sites and contemporary literature. En Just, E. y Wraggen, W. (Eds.), Adventurous Encounters (131-155). Cambridge: Cambridge Scholars University Press.

Roffe, J. T (2010). Multiplicity. En Parr, A (Ed.) The Deleuze Dictionary Revised Edition (181-182). Edinburgh: Edinburgh University Press.

Rogowska-Stangret, M. (2017) Corpor(e)al Cartographies of New Materialism: Meeting the Elsewhere Halfway. En The minnesota review 1 (88), 59-68.

Ruitenberg, C. W (2007) Here be dragons: Exploring cartography in educational theory and research. En Complicity: an international journal of complexity and education, 4(1), 7-24.

Schulte, C. M. (2016) Possible worlds: Deleuzian ontology and the project of listening in children’s drawing. En Cultural Studies-Critical Methodologies, 16(2), 141-150.

Souminen, A, Kallio-Tavin, M y Hernández-Hernández, F (2017) Arts-based research traditions and orientations in Europe: Perspectives from Finland and Spain. En Leavy, P. (Ed). Handbook of Arts-Based Research (101-120). Nueva York: Guildford Press.

Tiainen, M. y Parikka, J. (2013) The primacy of movement: variation, intermediality and biopolitics in Tero Saarinen's Hunt. En Barrett, E. y Bolt, B. (eds.) Carnal Knowledge: Towards a 'New Materialism' Through the Arts (205-224). Londres y Nueva York: I.B. Tauris.

Vidiella, J. (2012) Espacios y políticas culturales de la emoción. Pedagogías de contacto y prácticas de experimentación feministas. En Collados, A. y Rodrigo, J. (Coord.). Transductores: Pedagogías en red y prácticas instituyentes (62-84). Centro Jose GuerreroDiputación de Granada. Granada.

VV.AA. (2017) Investigación (educativa) basada en las artes. Materiales para el Mooc Tendencias emergentes en investigación educativa. La Laguna: Universidad de La Laguna. 
Hacia una pedagogía afectiva del movimiento / Towards an affective pedagogy of movement https://dx.doi.org/10.17561/rtc.n16.1 